\title{
Early onset versus late onset in Alzheimer's disease: What is the reliable cut-off?
}

\author{
Gianfranco Spalletta ${ }^{1 *}$, Vincenzo De Luca ${ }^{2}$, Alessandro Padovani $^{3}$, Luca Rozzini ${ }^{3}$, \\ Roberta Perri ${ }^{1}$, Amalia Bruni ${ }^{4}$, Vincenzo Canonico ${ }^{5}$, Alberto Trequattrini ${ }^{6}$, Giuseppe Bellelli ${ }^{7}$, \\ Carla Pettenati $^{8}$, Floriana Pazzelli ${ }^{1,9}$, Carlo Caltagirone ${ }^{1,10}$, Maria Donata Orfei ${ }^{1}$ \\ ${ }^{1}$ Department of Clinical and Behavioral Neurology, IRCCS Santa Lucia Foundation, Rome, Italy; \\ *Corresponding Author: g.spalletta@hsantalucia.it \\ ${ }^{2}$ Department of Psychiatry, University of Toronto, Toronto, Canada \\ ${ }^{3}$ Department of Neurology, University of Brescia, Brescia, Italy \\ ${ }^{4}$ Centro Regionale Di Neurogenetica, Azienda Sanitaria Provinciale Catanzaro, Lamezia Terme, Italy \\ ${ }^{5}$ Department of Geriatrics, University of Naples, Naples, Italy \\ ${ }^{6}$ Azienda Sanitaria Locale Città di Castello, Perugia, Italy \\ ${ }^{7}$ Department of Prevention and Clinical Medicine, University of Milano-Bicocca, Monza, Italy \\ ${ }^{8}$ Centro Regionale Alzheimer, Azienda Ospedaliera Guido Salvini, Passirana, Italy \\ ${ }^{9}$ NESMOS Department, Sapienza University of Rome, Rome, Italy \\ ${ }^{10}$ Department of Neuroscience, Tor Vergata University of Rome, Rome, Italy
}

Received 20 October 2012; revised 23 November 2012; accepted 3 December 2012

\section{ABSTRACT}

Objective: As the literature on conventional criteria for discriminating early-onset (EO) from late-onset (LO) Alzheimer's disease (AD) is sparse and controversial, the aim of this study was to establish a precise age at onset (AAO) criterion, by using a specific statistical procedure, and to describe the clinical characteristics of the two sub-groups. Methods: Admixture analysis was performed to establish the AAO cut-off in a multi-center study including 2000 AD patients consecutively recruited in eight Italian Memory Clinics. None of the patients were taking acetylcholinesterase inhibitors, antipsychotic or anti-depressant drugs. At the first diagnostic visit, they were administered the Mini Mental State Examination, the Basic and Instrumental Activities of Daily Living and the Neuropsychiatric Inventory to assess clinical phenomenology. Results: Using a specific statistical procedure, we established that AAO that discriminated EOfrom LO-AD was 66. Compared with the LO-AD group, the EO-AD group showed longer duration of illness and a higher educational level as well as less severe functional impairment and delusions. Conclusions: Differences in sociodemographic and clinical characteristics, such as duration of illness, education and delusion severity, suggested the involvement of different pathogenic processes. Additional studies are needed to further investigate the mechanisms underlying the disorder in the two sub-groups of $A D$ patients.

Keywords: Alzheimer's Disease; Onset;

Neuropsychiatric Symptoms; Admixture Analysis;

Pathogenesis; Cognitive Reserve

\section{INTRODUCTION}

In neurodegenerative diseases, age at onset (AAO) is an important factor in describing patients' clinical characteristics and may also be useful in selecting subgroups with more homogeneous pathogenic mechanisms and illness outcomes.

In Alzheimer's disease (AD) patients, AAO varies from 40 to 90 years. In diagnostic manuals [1], AD patients who develop symptoms before 65 years of age are considered as Early Onset (EO); most patients, however, are Late Onset (LO) [2].

In some studies, 65 years of age has been considered the arbitrary cut-off for AD [3]. However, unlike other neuropsychiatric conditions, such as obsessive-compulsive disorder [4], bipolar disorder [5] and schizophrenia [4-6], in AD age was established on a clinical basis with no real statistical foundation. This is not just a theoretical issue, because AAO could be an important criterion for exploring the clinical, neurobiological and genetic heterogeneity of $\mathrm{AD}$. In fact, it could allow clinicians to delineate homogenous phenotypes and confront different courses of illness more efficiently. Moreover, AAO might also contribute to facilitating the identification of 
different vulnerability genes or mutations of the same gene $[3,4]$. Thus, a more accurate classification is essential to more reliably discriminate AD sub-groups.

Furthermore, clinical data on EO- and LO-AD are sparse and controversial. In fact, although EO- and LOAD are sometimes considered clinically indistinguishable [2,7], results of some other works describe EO-AD as more functionally impaired than LO-AD or as more preserved $[8,9]$.

As the previous controversial data reported on this issue could have been due to limited sample size or sociodemographic and clinical heterogeneity, the main purpose of the present work was to use a specific statistical procedure to define a valid AAO cut-off for distinguishing EO- from LO-AD in a very large sample of drug-free AD patients at their first diagnostic assessment. A secondary aim of the study was to identify the clinical variables that distinguish EO- from LO-AD patients.

\section{METHODS}

\subsection{Subjects}

Subjects were consecutively recruited in eight Italian memory clinics, which were part of a multi-center study funded by the Italian Ministry of Health (MIS-RIFIDA). Inclusion criteria were: 1) diagnosis of probable $\mathrm{AD}$ according to the NINCDS-ADRDA criteria [10]; 2) first diagnostic assessment for dementia; 3) no acetylcholinesterase inhibitors (AChEI) and psychotropic drug (antidepressants or antipsychotics) treatment; and 4) availability of a reliable caregiver, defined as someone able to ensure the patient's compliance with assessment procedures and who contacted the patient at least twice weekly, with one contact being a personal visit. Exclusion criteria were: 1) major medical illness (non stabilized diabetes, obstructive pulmonary disease or asthma, hematologic/ oncologic disorders, vitamin B12 or folate deficiency, pernicious anemia, clinically significant and unstable active gastrointestinal, renal, hepatic, endocrine or cardiovascular disorders and recently treated hypothyroidism); 2) comorbidity of primary psychiatric or neurological disorders (e.g., schizophrenia, mood and/or anxiety disorders, stroke, Parkinson's disease, seizure disorder, head injury with loss of consciousness) or any other significant mental or neurological disorder other than AD; 3) drug/alcohol dependence and abuse; and 4) MRI or CT evidence of parenchyma abnormalities or neoplasm.

The study was approved by the local ethics committee. Consent was obtained after the nature and purposes of the study were explained to both patients and their caregivers.

\subsection{Diagnostic and Behavioral Assessment}

Trained physicians (neurologists, geriatricians and psychiatrists) made the diagnosis of $\mathrm{AD}$, and trained neuropsychologists made the cognitive and behavioral assessment.

Functional impairment was evaluated assessing basic (ADL) and instrumental (iADL) activities of daily living $[11,12]$. ADL include various activities regarding personal health and hygiene (e.g., eating, bathing, dressing and toileting), and $\mathrm{ADDL}$ include abilities that allow a person to live independently (e.g., food preparation, housekeeping and laundry, managing financial matters, shopping and using a telephone). When the ability is fully or at least partially preserved a score of 1 is assigned; when the ability has been lost a score of 0 is assigned. Thus, a total score ranging from 0 (total dependence) to 6 for ADL or 8 for iADL (total independence) is obtained.

We also used the Clinical Dementia Rating Scale (CDR) [13] because it characterizes six domains of cognitive and functional performance: memory, orientation, judgment and problem solving, community affairs, home and hobbies and personal care. The information needed to make each rating is obtained through a semi-structured interview with a reliable caregiver. In addition to ratings on a 5-point scale for each domain (except personal care, which is rated on a 4-point scale), an overall CDR score is derived by standard algorithm. This score is useful to globally stage the level of impairment as follows: $0=$ no impairment, $0.5,1,2$ and 3 indicate very mild, mild, moderate and severe dementia.

Patients were administered the Mini-Mental State Examination (MMSE) [14] to assess global cognitive level. This is a widely used neurocognitive screening test, which measures orientation, language, verbal memory, attention, visuospatial function and mental control. It includes 16 items; scores range from 30 (no impairment) to 0 (maximum impairment).

The Neuropsychiatric Inventory (NPI) [15] was used to measure behavioral symptoms. It evaluates 12 neuropsychiatric symptoms that are common in dementia: delusions, hallucinations, agitation/aggression, dysphoria/ depression, anxiety, euphoria/elation, apathy/indifference, disinhibition, irritability/lability, aberrant motor behaviors (AMB), nighttime behavioral disturbances and appetite/eating disturbances. An informant rates the frequency and severity of each of these dimensions; the final score is obtained by multiplying the first two scores. In each domain, NPI presents a screening question. If it is answered negatively, the interviewer moves to the next domain. If it is answered affirmatively, specific neuropsychiatric symptoms are assessed within that domain. If any of these symptoms are presented, they are rated on a 4-point frequency scale and a separate 3-point severity scale. The NPI also assesses the amount of caregiver distress engendered by each neuropsychiatric symptom 
on a 5-point scale. Thus, the score for each dimension ranges from 0 to 12 , with a maximum total score of 144 in the 12-item version.

\subsection{Statistical Analyses}

AAO was defined as the age when cognitive symptoms of memory failure and deficiency in at least one other cognitive domain first appeared, interfering with the patient's social or occupational functioning, which was assessed by the clinician's interview with a reliable caregiver. In particular, the primary caregiver was asked to describe the "first problem", and to provide detailed information about it, to further identify the "earliest episode" by providing a calendar date, to clarify any ambiguities and to establish a "symptom-free date" representing the last time no symptoms of dementia were presented.

Admixture analysis was used to determine the best fitting theoretical model for the observed distribution of AAO. In order to choose the best fitting model, we applied the $\chi^{2}$ goodness of fit test using $\chi^{2}$ fit command implemented in the denormix module of the software STATA (release 8, StataCorp, College Station, TX). The best fitting model was chosen based on the highest p-value of the $\chi^{2}$ test, which indicated that the theoretical model did not deviate from the empirical distribution function of AAO in our sample. The theoretical AAO function defined the AAO probability density across different ages. Each patient's probability of belonging to each AAO subgroup was calculated using the theoretical AAO function. Patients were then assigned to the distribution they had the highest probability of belonging to. The theoretical AAO function was used to calculate these probabilities and to locate cut-off points. This method was previously used to define AAO in patients with obsessive-compulsive disorder, bipolar-I disorder and schizophrenia [4-6,16].

A series of unpaired t-tests were performed to compare age, age at onset, illness duration from onset of symptoms to the first diagnostic visit, educational level, ADL and iADL, CDR, MMSE and the mean scores of each NPI symptom of the EO-AD group with those of the LO-AD group. Differences were considered significant if $\mathrm{p}<$ 0.05 . Neuropsychiatric symptom significant differences were adjusted ( $p<0.05 / 12$, $p<0.0042$ after the Bonferroni correction for multiple comparisons). The comparison between EO- and LO-AD with respect to the gender categorical variable was made using the chi-square test, with a statistical significance level of $\mathrm{p}<0.05$.

\section{RESULTS}

A consecutive sample of 2000 AD patients (604 males, 1396 females) was included in the analysis. The mean
$( \pm \mathrm{SD})$ age at the first clinical contact for diagnostic purpose was $75.4( \pm 7.29)$ years. The overall mean $( \pm \mathrm{SD})$ AAO was $73.02( \pm 7.40)$ years. The socio-demographic and clinical characteristics of the study sample are described in Table 1.

Admixture analysis yielded a combination of two normal theoretical distributions. The EO subgroup represented $24.7 \%$ of the sample. The two subgroups were divided by AAO, with 66 years of age as an ideal cut-off characterized by the point at which the two curves intersected (see Figure 1). Thus, patients with an AAO $\leq 66$ years were considered included in the EO group and those with an AAO > 66 years were included in the LO group. At age 66 the probability of belonging to the EO subgroup was $54 \%$ and of belonging to the LO subgroup, $46 \%$.

As expected by the sub-group selection, the EO-AD patients were younger and had a higher educational level than the LO-AD patients. The EO-AD group included significantly more males $(41 \%)$ than the LO-AD group $(28 \%)$ (chi-square $=20.903 ; \mathrm{df}=1 ; \mathrm{p}<0.0001)$. Also, the EO-AD patients had a significantly longer duration of illness from AD symptom onset to the first diagnostic visit, were less impaired on both the ADL and the $\mathrm{AADL}$, and performed worse than the LO-AD group on the CDR.

With regard to the NPI scores, at the uncorrected $(\mathrm{p}<$ 0.05) statistical level the LO-AD group showed significantly higher symptom severity for Delusions, Agitation and Nighttime Behavioral Disturbances than the EO-AD group. However, at the corrected $(p<0.0042)$ level, only the differences related to delusion symptom severity survived.

\section{CONCLUSION}

The main aim of this study was to establish a valid cut-off age for discriminating EO-AD from LO-AD. For this purpose, we applied admixture analysis to investigate a large sample of AD patients. This is a specific statistical procedure that has been widely used to determine cut-off ages in other neuropsychiatric disorders [4-6]. We also explored the sociodemographic and clinical features of the two emerging AD subgroups.

Five main results emerged. First, the main finding of our study was the identification of two clinically relevant Gaussian curves in the observed distribution of AAO. The age 66 cut-off seems to separate the sample into two identifiable AD subtypes and only differs slightly from the previous conventionally used cut-off of 65 years [1]. Considering the interaction between AAO and neuropsychological, neuropsychiatric, genetic and neuroanatomic factors, the confirmation that homogeneous EO- and LO-AD subgroups exist should provide a good basis for future studies aimed at clarifying the mechanisms of AD. 


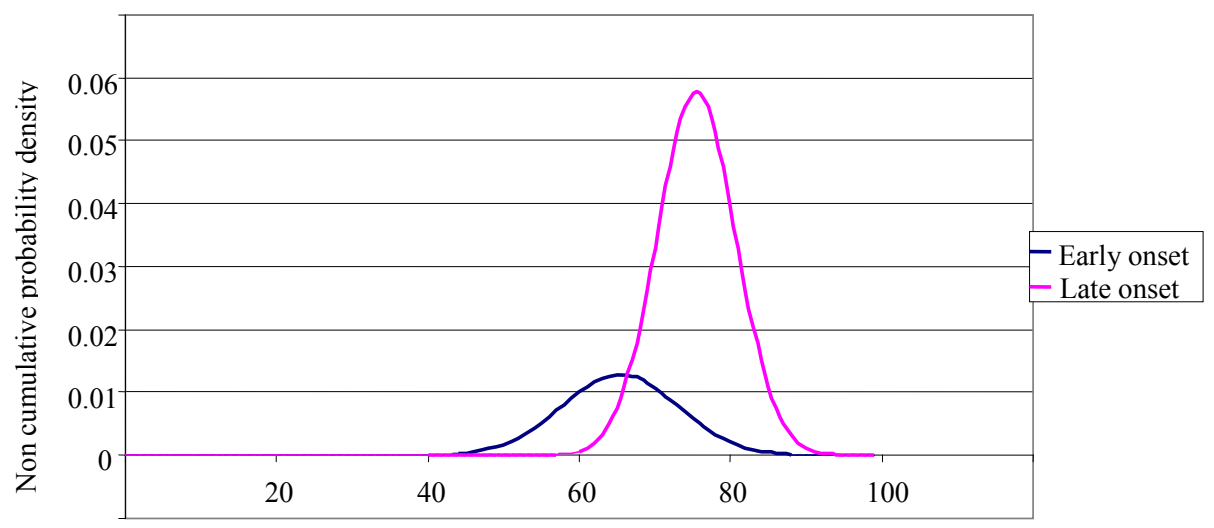

Age at Onset (AAO)

Figure 1. Age at onset probability density in early onset and late onset AD.

Table 1. Socio-demographic and clinical characteristics of 346 early onset AD patients compared with 1654 late onset AD patients.

\begin{tabular}{|c|c|c|c|}
\hline Characteristics & Early Onset AD Mean $( \pm$ SD) & Late Onset AD Mean $( \pm$ SD $)$ & t-value (p) \\
\hline Age (years) & $63.777(5.187)$ & $77.895(4.915)$ & $-48.115(<0.0001)$ \\
\hline Age at Onset (years) & $60.873(4.791)$ & $75.568(4.923)$ & $-50.720(<0.0001)$ \\
\hline Illness Duration (years) & $2.905(2.110)$ & $2.327(1.811)$ & $5.236(<0.0001)$ \\
\hline Educational Level (years) & $7.494(3.917)$ & $6.281(3.806)$ & $5.364(<0.0001)$ \\
\hline ADL & $5.315(1.261)$ & $5.093(1.343)$ & $2.832(0.0047)$ \\
\hline IADL & $4.705(2.402)$ & $4.099(2.216)$ & $4.558(<0.0001)$ \\
\hline CDR & $1.213(0.730)$ & $1.329(0.660)$ & $-2.534(0.0114)$ \\
\hline MMSE & $19.260(5.578)$ & $18.773(5.235)$ & $1.557(0.1196)$ \\
\hline NPI Delusions & $0.650(1.974)$ & $1.140(2.442)$ & $-3.501(0.0005)^{* *}$ \\
\hline NPI Hallucinations & $0.399(1.686)$ & $0.599(1.776)$ & $-1.924(0.0545)$ \\
\hline NPI Agitation & $1.289(2.232)$ & $1.690(2.640)$ & $-2.638(0.0084)^{*}$ \\
\hline NPI Depression/Dysphoria & $2.526(2.778)$ & $2.624(2.966)$ & $-0.565(0.5724)$ \\
\hline NPI Anxiety & $2.075(2.639)$ & $2.186(2.873)$ & $-0.663(0.5074)$ \\
\hline NPI Euphoria & $0.439(1.472)$ & $0.388(1.281)$ & $0.658(0.5108)$ \\
\hline NPI Apathy & $3.199(3.400)$ & $3.215(3.466)$ & $-0.077(0.9383)$ \\
\hline NPI Disinhibition & $0.581(1.783)$ & $0.677(1.846)$ & $-0.881(0.3782)$ \\
\hline NPI Irritability & $1.624(2.528)$ & $1.774(2.667)$ & $-0.961(0.3366)$ \\
\hline NPI Aberrant Motor Behavior & $1.318(2.642)$ & $1.567(2.923)$ & $-1.465(0.1430)$ \\
\hline NPI Nighttime Behavioral Disturbances & $1.011(2.031)$ & $1.342(2.596)$ & $-2.022(0.0433)^{*}$ \\
\hline NPI Appetite/Eating Disturbances & $1.046(2.288)$ & $1.323(2.583)$ & $-1.670(0.0951)$ \\
\hline NPI Total & $16.707(14.412)$ & $18.942(16.246)$ & $-2.147(0.0320)^{*}$ \\
\hline
\end{tabular}

Note: $\mathrm{AD}=$ Alzheimer's disease, $\mathrm{SD}=$ Standard Deviation, ADL = Activities of Daily Living, IADL = Instrumental Activities of Daily Living, CDR = Clinical Dementia Rating, MMSE = Mini-Mental State Examination, NPI = Neuropsychiatric Inventory. Significant values are highlighted in bold for all variables. For NPI scores values were: ${ }^{*}$ significant at the uncorrected level $(\mathrm{p}<0.05)$; and ${ }^{* *}$ significant at the corrected level $(\mathrm{p}<0.0042$, after Bonferroni correction for multiple comparisons). 
Second, with regard to sociodemographic characteristics, as expected from the sample selection we found that the EO-AD patients were younger and had a higher educational level than the LO-AD patients. This finding was expected because of increasing access to the educational system in Italy over the past century, especially after the Second World War, thanks to laws aimed at countering illiteracy and growing economic prosperity. Thus, younger people are more likely to have a higher educational level.

Third, the EO-AD group included significantly fewer females than the LO-AD group. This finding supports the hypothesis that EO- and LO-AD have different genetic underpinnings. In fact, a strong association between the apolipoprotein E4 allele (ApoE4), which is notoriously linked to cognitive impairment and decline [17], and female gender has been frequently described $[18,19]$. Analogously, the gene for the angiotensin converting enzyme (ACE), which has recently been reported to be associated with risk of $\mathrm{AD}$, showed a gender-specific association of ACE genotype with $\mathrm{AD}$ in the female clinical population [20]. These studies suggest that gender may interact with genetic factors to differentially influence risk factors and pathogenic mechanisms in AD. More specifically, our data suggest that gender may determine a different pathogenesis in EO- and LO-AD. However, although some previous studies also found that male gender was associated with EO-AD [21], other works show either no difference or a female predominance in EO-AD $[8,22,23]$. This discrepancy in gender frequency among different studies could be due to the different methodologies used, such as choice of sample size or a retrospective approach versus consecutive recruitment of first diagnosis patients who are, therefore, very near to the illness onset. In any case, this issue certainly requires further investigation.

Fourth, EO-AD subjects tended to be less functionally impaired than LO-AD subjects, although no difference in MMSE score emerged between the two groups. One very challenging comprehensive explanation that would link all of these results, is that cognitive reserve has a role in protecting against cognitive impairment. Indeed, cognitive reserve refers to selective micro- and macrostructural brain changes in gray matter, enabling the individual to recruit compensatory mechanisms to combat damaging pathological reactions and delay functional impact and AD symptom expression [24,25]. Cognitive reserve is significantly correlated with educational level, which plays a fundamental role in mediating reserve against degenerative processes, particularly with regard to hippocampal microstructure [26]. Thus, we could hypothesize that our EO-AD patients, who were more educated and therefore had a greater cognitive reserve than our LO-AD patients, had to have more severe pathological changes before they exhibited cognitive deterioration
[27]. Indeed, previous studies have highlighted a greater neuropathological burden in EO-AD than in LO-AD [2], in particular, the presence of more neuritic plaques (NPs), neurofibrillary tangles (NFTs), and non stroke subcortical ischemic lesions [27], which would support our hypothesis. Interestingly, EO-AD participants also showed a longer duration of illness from $\mathrm{AD}$ symptom onset to the first actual diagnosis of AD than LO-AD participants. An intriguing hypothesis is that in EO cognitive reserve lengthens the prodromal phase of the illness, preventing and thus delaying the definite decline toward the actual diagnosis of $\mathrm{AD}[25,28]$. In any case, this hypothesis requires further investigation. A more definite fact, however, is that the increased severity of delusions and, more generally, the increased severity of neuropsychiatric symptoms in LO-AD may lead to an earlier first visit assessment because of the heavy caregiver burden caused by behavioral symptoms.

Fifth, in our study the EO-AD patients suffered from lower severity of delusions than the LO-AD patients. Further, the latter group tended to suffer from some additional behavioral symptoms, namely, agitation and nighttime disturbances, as already described in a previous study [8]. There may be various explanations for this. First, older age and female gender, both characteristic of our LO sample, are frequently described as factors independently associated with the presence of psychotic symptoms, especially delusions [29]. This also supports the hypothesis of different mechanisms in EO and LO subjects. Second, although cognitive functions are greatly affected by subcortical vascular lesions, which are quite frequent in EO subjects [30,31], some behavioral domains may be less involved. In fact, severity of symptoms of depression and apathy may be increased by vascular lesions in the frontal-subcortical circuit or the cingulated areas [32,33], whereas psychotic symptoms are associated with hypometabolism and hypoperfusion in the fronto-parieto-temporal areas [25,34,35]. Thus, EO and LO patients may also differ with respect to neuroanatomical pathogenic factors that produce different behavioral symptoms. Finally, we can hypothesize that, MMSE score being equal, cognitive reserve buffers functional and behavioral deficits in EO-AD subjects, which thus appear more severe in LO patients.

Before concluding, we would like to highlight the strengths of our study. We investigated a large cohort of first diagnosis, drug-free, systematically evaluated subjects and used validated probabilistic tools to assign each subject to the appropriate sub-group (i.e. EO or LO AD). Thus, our cut-off points were valid.

Some issues that may limit our study results must be also described. First, our sample was homogeneous with regard to ethnicity (all participants were white, Caucasian Italians) and our data were cross-sectional. Thus, in 
future studies our results should be confirmed in other populations and should consider the longitudinal course of behavioral and neuropsychological clinical phenomenology in the EO- and LO-AD groups. Furthermore, we considered only the MMSE score as a cognitive index. Indeed, some previous studies reported that EO-AD is characterized by a more rapid progression of cognitive impairment, particularly in specific domains such as naming abilities, attentional and visuo-spatial dysfunctions [36-38]. However, these works included smaller samples than ours and relatively limited test batteries. Therefore, future studies should try to define a more precise neuropsychological profile of the two AD subgroups. Lastly, in our study we included only drug-free patients at their first diagnostic visit in order to avoid the influence of drug treatment on symptom expression and to reduce effects on different phases of the illness. In any case, this issue certainly requires further investigation, also in longitudinal studies.

In conclusion, our study using admixture analysis (a statistical procedure validated to discriminate people with homogeneous AAO) established 66 years of age as the new cut-off for discriminating EO- from LO-AD. The existence of two AAO AD subgroups should be further confirmed by means of external validators, namely, genetic, neuroanatomical, cognitive, biochemical or neurophysiological probes. In fact, an accurate and timely diagnosis and a more precise understanding of the clinical features of EO-AD with respect to LO-AD is required in order to gather reliable epidemiological data, highlight risk factors, explain pathogenic mechanisms and develop more efficient therapeutic interventions. In particular, given the devastating nature of early-onset dementias, these findings emphasize the need to evaluate EO-AD patients to find efficacious methods of prevention and treatment.

Conflicts of Interest and Source of Funding: This research was funded by Italian Ministry of Health RC0607-08-09/A grants. No conflict of interest to declare.

\section{REFERENCES}

[1] APA (2000) Diagnostic and Statistical Manual of Mental Disorder, DSM-IV-TR. Washington. http://www.apa.org/

[2] Reitz, C., Brayne, C. and Mayeux, R. (2011) Epidemiology of Alzheimer disease. Nature Reviews. Neurology, 7, 137-152. doi:10.1038/nrneurol.2011.2

[3] Van der Flier, W.M., Pijnenburg, Y.A., Fox, N.C. and Scheltens, P. (2010) Early-onset versus late-onset Alzheimer's disease: The case of the missing APOE varepsilon4 allele. The Lancet Neurology, 10, 280-288. doi:10.1016/S1474-4422(10)70306-9

[4] Delorme, R., Golmard, J.L., Chabane, N., Millet, B., Krebs, M.O., Mouren-Simeoni, M.C. and Leboyer, M. (2005) Admixture analysis of age at onset in obsessive-compul- sive disorder. Psychological Medicine, 35, 237-243. doi: $10.1017 / \mathrm{S} 0033291704003253$

[5] Hamshere, M.L., Gordon-Smith, K., Forty, L., Jones, L., Caesar, S., Fraser, C., Hyde, S., Tredget, J., Kirov, G., Jones, I., Craddock, N. and Smith, D.J. (2009) Age-atonset in bipolar-I disorder: Mixture analysis of 1369 cases identifies three distinct clinical sub-groups. Journal of Affective Disorders, 116, 23-29. doi:10.1016/j.jad.2008.10.021

[6] Panariello, F., O’Driscoll, L., De Souza, R.P., Manchia, A. M., Kennedy, J. and De Luca, V. (2010) Age at onset in Canadian schizophrenia patients: Admixture analysis. Schizophrenia Research, 122, 278-279.

doi:10.1016/j.schres.2009.10.024

[7] Licht, E.A., McMurtray, A.M., Saul, R.E. and Mendez, M.F. (2007) Cognitive differences between early- and late-onset Alzheimer's disease. American Journal of Alzheimer's Disease and Other Dementias, 22, 218-222. doi:10.1177/1533317506299156

[8] Toyota, Y., Ikeda, M., Shinagawa, S., Matsumoto, T., Matsumoto, N., Hokoishi, K., Fukuhara, R., Ishikawa, T., Mori, T., Adachi, H., Komori, K. and Tanabe, H. (2007) Comparison of behavioral and psychological symptoms in early-onset and late-onset Alzheimer's disease. International Journal of Geriatric Psychiatry, 22, 896-901. doi:10.1002/gps.1760

[9] Van der Vlies, A.E., Koedam, E.L., Pijnenburg, Y.A., Twisk, J.W., Scheltens, P. and Van der Flier, W.M. (2009) Most rapid cognitive decline in APOE epsilon4 negative Alzheimer's disease with early onset. Psychological Medicine, 39, 1907-1911. doi:10.1017/S0033291709005492

[10] McKhann, G., Drachman, D., Folstein, M., Katzman, R., Price, D. and Stadlan, E.M. (1984) Clinical diagnosis of Alzheimer's disease: Report of the NINCDS-ADRDA Work Group under the auspices of Department of Health and Human Services Task Force on Alzheimer's Disease. Neurology, 34, 939-944. doi:10.1212/WNL.34.7.939

[11] Katz, S., Down, T., Cash, H. and Grotz, R. (1970) Progress in the development of the index of ADL. The Gerontologist, 10, 20-30. doi:10.1093/geront/10.1_Part_1.20

[12] Lawton, M. and Brody, E. (1969) Assessment of older people: Self-maintaining and instrumental activities of daily living. Gerontologist, 9, 179-186. doi:10.1093/geront/9.3 Part 1.179

[13] Morris, J. (1993) The Clinical Dementia Rating (CDR): Current version and scoring rules. Neurology, 43, 24122414. doi:10.1212/WNL.43.11.2412-a

[14] Folstein, M.F., Folstein, S.E. and McHugh, P.R. (1975) "Mini-mental state". A practical method for grading the cognitive state of patients for the clinician. Journal of Psychiatric Research, 12, 189-198. doi:10.1016/0022-3956(75)90026-6

[15] Cummings, J.L. (1997) The neuropsychiatric inventory: Assessing psychopathology in dementia patients. Neurology, 48, S10-S16. doi:10.1212/WNL.48.5_Suppl_6.10S

[16] Tozzi, F., Manchia, M., Galwey, N.W., Severino, G., Del Zompo, M., Day, R., Matthews, K., Strauss, J., Kennedy, 
J.L., McGuffin, P., Vincent, J.B., Farmer, A. and Muglia, P. (2011) Admixture analysis of age at onset in bipolar disorder. Psychiatry Research, 185, 27-32. doi:10.1016/i.psychres.2009.11.025

[17] Small, B.J., Graves, A.B., McEvoy, C.L., Crawford, F.C., Mullan, M. and Mortimer, J.A. (2000) Is APOE-epsilon4 a risk factor for cognitive impairment in normal aging? Neurology, 54, 2082-2088. doi:10.1212/WNL.54.11.2082

[18] Beydoun, M.A., Boueiz, A., Abougergi, M.S., KitnerTriolo, M.H., Beydoun, H.A., Resnick, S.M., O’Brien, R. and Zonderman, A.B. (2010) Sex differences in the association of the apolipoprotein E epsilon 4 allele with incidence of dementia, cognitive impairment, and decline. Neurobiology of Aging, 33, 720-731. doi:10.1016/j.neurobiolaging.2010.05.017

[19] Giannattasio, C., Poleggi, A., Puopolo, M., Pocchiari, M., Antuono, P., Dal Forno, G., Wekstein D.R., Matera, M.G., Seripa, D., Acciarri, A., Bizzarro, A., Lauria, A. and Masullo, C. (2008) Survival in Alzheimer's disease is shorter in women carrying heterozygosity at codon 129 of the PRNP gene and no APOE epsilon 4 allele. Dementia and Geriatric Cognitive Disorders, 25, 354-358. doi:10.1159/000119730

[20] Crawford, F., Abdullah, L., Schinka, J., Suo, Z., Gold, M., Duara, R. and Mullan, M. (2000) Gender-specific association of the angiotensin converting enzyme gene with Alzheimer's disease. Neuroscience Letters, 280, 215-219. doi:10.1016/S0304-3940(00)00791-6

[21] Picard, C., Pasquier, F., Martinaud, O., Hannequin, D. and Godefroy, O. (2010) Early onset dementia: Characteristics in a large cohort from academic memory clinics. Alzheimer Disease and Associated Disorders, 25, 203-205. doi:10.1097/WAD.0b013e3182056be7

[22] Newens, A., Forster, D., Kay, D., Kirkup, W., Bates, D. and Edwardson, J. (1993) Clinically diagnosed presenile dementia of the Alzheimer type in the Northern Health Region: Ascertainment, prevalence, incidence and survival. Psychological Medicine, 23, 631-644. doi:10.1017/S0033291700025411

[23] Kokmen, E., Chandra, V. and Schoenberg, B.S. (1988) Trends in incidence of dementing illness in Rochester, Minnesota, in three quinquennial periods, 1960-1974. Neurology, 38, 975-980. doi:10.1212/WNL.38.6.975

[24] Fairjones, S.E., Vuletich, E.J., Pestell, C. and Panegyres, P.K. (2011) Exploring the role of cognitive reserve in earlyonset dementia. American Journal of Alzheimer's Disease and Other Dementias, 26, 139-144. doi:10.1177/1533317510397328

[25] Serra, L., Cercignani, M., Petrosini, L., Basile, B., Perri, R., Fadda, L., Spano, B., Marra, C., Giubilei, F., Carlesimo, G.A., Caltagirone, C. and Bozzali, M. (2011) Neuroanatomical correlates of cognitive reserve in Alzheimer disease. Rejuvenation Research, 14, 143-151. doi:10.1089/rej.2010.1103

[26] Piras F., Cherubini, A., Caltagirone, C. and Spalletta, G. (2011) Education mediates microstructural changes in bilateral hippocampus. Human Brain Mapping, 32, 282-289. doi:10.1002/hbm.21018

[27] Marshall, G.A., Fairbanks, L.A., Tekin, S., Vinters, H.V. and Cummings, J.L. (2007) Early-onset Alzheimer's disease is associated with greater pathologic burden. Journal of Geriatrics Psychiatry and Neurology, 20, 29-33. doi:10.1177/0891988706297086

[28] Mortimer, J.A., Borenstein, A.R., Gosche, K.M. and Snowdon, D.A. (2005) Very early detection of Alzheimer neuropathology and the role of brain reserve in modifying its clinical expression. Journal of Geriatrics Psychiatry and Neurology, 18, 218-223.

doi:10.1177/0891988705281869

[29] Hirono, N., Mori, E., Yasuda, M., Ikejiri, Y., Imamura, T., Shimomura, T., Ikeda, Hashimoto, M.M. and Yamashita, H. (1998) Factors associated with psychotic symptoms in Alzheimer's disease. Journal of Neurology, Neurosurgery and Psychiatry, 64, 648-652. doi:10.1136/jnnp.64.5.648

[30] Esiri, M.M., Nagy, Z., Smith, M.Z., Barnetson, L. and Smith, A.D. (1999) Cerebrovascular disease and threshold for dementia in the early stages of Alzheimer's disease. Lancet, 354, 919-920. doi:10.1016/S0140-6736(99)02355-7

[31] Geppert, A.M., Wroblewska, K.A. and Przedpelska-Ober, E.M. (2007) Greater frequency of subcortical lesions in severely demented patients with early onset Alzheimer's disease. Alzheimer's \& Dementia, 3, 54-57. doi:10.1016/j.jalz.2006.10.007

[32] Bella, R., Pennisi, G., Cantone, M., Palermo, F., Pennisi, M., Lanza, G., Zappia, M. and Paolucci, S. (2010) Clinical presentation and outcome of geriatric depression in subcortical ischemic vascular disease. Gerontology, 56, 298-302. doi:10.1159/000272003

[33] Lavretsky, H., Zheng, L., Weiner, M.W., Mungas, D., Reed, B., Kramer, J.H., Jagust, W., Chui, H. and Mack, W. J. (2008) The MRI brain correlates of depressed mood, anhedonia, apathy, and anergia in older adults with and without cognitive impairment or dementia. International Journal of Geriatric Psychiatry, 23, 1040-1050. doi: $10.1002 / \mathrm{gps} .2030$

[34] Mentis, M.J., Weinstein, E.A., Horwitz, B., McIntosh, A. R., Pietrini, P., Alexander, G.E., Furey, M. and Murphy, D. G. (1995) Abnormal brain glucose metabolism in the delusional misidentification syndromes: A positron emission tomography study in Alzheimer disease. Biological Psychiatry, 38, 438-449. doi:10.1016/0006-3223(94)00326-X

[35] Staff, R.T., Shanks, M.F., Macintosh, L., Pestell, S.J., Gemmell, H.G. and Venneri, A. (1999) Delusions in Alzheimer's disease: Spet evidence of right hemispheric dysfunction. Cortex, 35, 549-560. doi:10.1016/S0010-9452(08)70818-9

[36] Fujimori, M., Imamura, T., Yamashita, H., Hirono, N., Ikejiri, Y., Shimomura, T. and Mori, E. (1998) Age at onset and visuocognitive disturbances in Alzheimer disease. Alzheimer Disease and Associated Disorders, 12, 163166. doi:10.1097/00002093-199809000-00007

[37] Imamura, T., Takatsuki, Y., Fujimori, M., Hirono, N., Ikejiri, Y., Shimomura, T., Hashimoto, M., Yamashita, H. and Mori, E. (1998) Age at onset and language disturbances in Alzheimer's disease. Neuropsychologia, 36, 945-949. doi:10.1016/S0028-3932(98)00010-4 
[38] Jacobs, D., Sano, M., Marder, K., Bell, K., Bylsma, F., Lafleche, G., Albert, M., Brandt, J. and Stern, Y. (1994) Age at onset of Alzheimer's disease: Relation to pattern of cognitive dysfunction and rate of decline. Neurology, 44, 1215-1220. doi:10.1212/WNL.44.7.1215 